\title{
Pre-exposure prophylaxis for infants exposed to HIV through breast feeding
}

Philippe Van de Perre and colleagues say current strategies for preventing transmission of HIV infection from mother to child are inadequate and call for infants to be given pre-exposure prophylaxis

\author{
Philippe Van de Perre professor ${ }^{12}$, Chipepo Kankasa doctor ${ }^{3}$, Nicolas Nagot professor $^{12}$, Nicolas \\ Meda professor ${ }^{4}$, James K Tumwine professor ${ }^{6}$, Anna Coutsoudis professor ${ }^{7}$, Thorkild Tylleskär \\ professor $^{8}$, Hoosen Coovadia professor ${ }^{9}$
}

${ }^{1}$ Pathogenesis and Control of Chronic Infections, UMR 1058 INSERM, University of Montpellier, France; ${ }^{2} \mathrm{CHU}$ Montpellier, Montpellier, France; ${ }^{3}$ Department of Paediatrics and Child Health, University Teaching Hospital, Lusaka, Zambia; ${ }^{4}$ Centre Muraz, Bobo-Dioulasso, Burkina Faso; ${ }^{5} \mathrm{Centre}$ of International Research for Health, Faculty of Health Sciences, University of Ouagadougou, Ouagadougou, Burkina Faso; ${ }^{6}$ Department of Paediatrics and Child Health, College of Health Sciences, Makerere University, Kampala, Uganda; ${ }^{7}$ Department of Paediatrics and Child Health, University of KwaZulu-Natal, Durban, South Africa; ${ }^{8}$ Centre for International Health, University of Bergen, Bergen, Norway; ${ }^{9}$ Match Health Systems, School of Public Health, University of the Witwatersrand, South Africa.

The AIDS 2016 conference, held in July in Durban, South Africa, lauded pre-exposure prophylaxis (PrEP) as the way forward for substantially reducing the rate of new HIV infections worldwide. PrEP is defined as the continuous or intermittent use of an antiretroviral drug or drug combination to prevent HIV infection in people exposed to the virus. The underlying pathophysiological rationale is that impregnating uninfected cells and tissues with an antiviral drug could prevent infection by both cell-free and cell-associated HIV (cell-to-cell transfer). PrEP's tolerance and efficacy have been demonstrated in well designed clinical trials in men who have sex with men (MSM). ${ }^{12}$ In the Ipergay trial, $86 \%$ of HIV infections were averted in highly exposed men. ${ }^{2}$ PrEP has also been evaluated in other highly exposed groups such as transgender women, injecting drug users, serodiscordant heterosexual couples, and commercial sex workers. ${ }^{3}$

\section{HIV exposed children: lost in translation}

Uninfected pregnant or breastfeeding women in high incidence areas have also been suggested as a potential target population for PrEP, but infants exposed to HIV through breast feeding have not been mentioned. ${ }^{4}$ Numerous public declarations and petitions have produced a strong advocacy for extension of the PrEP principle to all high risk populations exposed to HIV, considering access to PrEP as part of human rights. Recently, the World Health Organization recommended offering PrEP to any population in which the expected incidence of HIV infection is above 3 per 100 person-years. ${ }^{35}$ So why are breastfed infants born to HIV infected women, a population that often has an overall HIV acquisition rate above 3/100 person-years, not receiving this clearly beneficial preventive health measure?

\section{Current strategy not good enough}

Since June 2013, the WHO has recommended universal lifelong antiretroviral therapy (ART) — known as "option B+" - for all pregnant and breastfeeding women infected with HIV-1, with the objective of eliminating mother-to-child transmission (defined by $\mathrm{WHO}$ as an overall rate of transmission lower than $5 \%) .{ }^{5}$ The $\mathrm{B}+$ strategy also recommends that their babies receive nevirapine for six weeks to mitigate the risks of transmission during delivery. But infants who are breast fed continue to be exposed to a substantial risk of infection beyond the six week prophylaxis period.

The B+ strategy has been rolled out in most programmes to prevent mother-to-child transmission worldwide without any additional protection for breastfed infants. Although these programmes have been shown to increase the number of pregnant and breastfeeding women who receive ART, their success in prevention of infection in infants is less clear. According to UNAIDS estimates, improvement in services to prevent mother-to-child HIV transmission since 2010 has reduced the annual number of new infections among children globally by $56 \%{ }^{6}$ However, the few available programmatic data on long term residual HIV transmission rates suggest that this is mainly accounted for by reduced in utero and intrapartum HIV transmission rather than in postnatal transmission through breast feeding. Also, there is considerable variation across countries and continents, with many countries, mainly in Africa 
and Asia, seeing no change in HIV incidence among children. An update of the UNAIDS 2015 report suggests that in 2015 the average mother-to-child transmission rate was $8.9 \%$ among 21 Global Plan African countries, and only five of these countries - Namibia, Uganda, Swaziland, Botswana and South Africa-have reached the target transmission rate of below $5 \%{ }^{7}$

\section{Reasons for continued transmission}

Most of the residual transmission is attributable to exposure through breast feeding. A recent study assessed community viral load in Kenyan, Malawian, and South African households, including more than 11000 women of child bearing age, of whom 3296 were pregnant or breast feeding. A total of 608 pregnant or breastfeeding women had HIV infection, with the proportion with plasma RNA above 1000 copies/ml varying from $27 \%$ in Malawi to $73 \%$ in Kenya. ${ }^{8}$ Some of the women who had detectable viral load were unaware of their infection because they had not been tested or had become infected after antenatal screening; others had not started ART or were not taking it as recommended.

In 2015, about 150000 children were infected with HIV worldwide. The vertical transmission rate from mother-to-infant at six weeks was $5 \%$ but rose to $8.9 \%$ by the conclusion of breast feeding. ${ }^{7}$ In Africa, the reasons for this high residual burden of child infections are multiple. The main reason is operational, with challenges in all phases of the care cascade (test, treat, and retain in care), including consistent testing of HIV exposed infants, starting infants on treatment, and retaining infants in care.

Primary obstacles to linkage and retention include the distance and resources required to travel to a health facility, cultural or stigma related challenges, logistic hurdles that exist in antenatal care centres, and resources and efficacy of linkage to definitive HIV care. Observational studies in different African settings report less than optimal adherence, with only $50-70 \%$ viral suppression in women one year after starting ART. In Malawi, where the B+ strategy was rolled out in 2011, one fifth of women identified never started ART during the early phases of the programme. ${ }^{9}$ In the early phases of the Swaziland programme, postnatal retention in care for HIV infected women was only $37 \%$ overall and $50 \%$ for those who started ART during pregnancy. ${ }^{10}$

A study in Malawi found that women who started ART to prevent transmission to their child were five times more likely to default than women who started treatment for their own health. ${ }^{9}$ Maternal discontinuation of ART while breast feeding considerably increases risk of HIV transmission to the infant because of viral rebound, as observed after interrupting maternal zidovudine prophylaxis in the DITRAME study. ${ }^{11}$

Furthermore, cell-to-cell transfer of HIV is not inhibited in mothers taking ART in many cases. ${ }^{12}$ The residual postnatal transmission rate from a mother with an ART suppressed viral load has been estimated at $0.2 \%$ per month of breastfeeding. This corresponds to an expected residual rate of $2.4 \%$ at 12 months. ${ }^{13}$ Since the latest WHO/Unicef guidelines for HIV and infant feeding recommend 24 months of breast feeding rather than $12,{ }^{14}$ the duration of infant HIV exposure will be much extended, increasing the risk of additional HIV infections.

\section{Infant prophylaxis has been used before}

Administration of a daily antiviral drug to an uninfected but exposed breastfed infant meets the definition of PrEP: the prophylaxis is administered before exposure (ideally from birth) to an uninfected infant whose exposure to HIV is intermittent (during breast feeding) and persistent. Ironically, infants born to HIV infected women were the first to participate in ARV prophylaxis trials. They have probably contributed the highest number of participants in such studies worldwide. Indeed, prophylaxis with oral zidovudine was integrated in the first prophylactic protocol (ACTG 076) reported in 1994. ${ }^{15}$ Thereafter, numerous trials have included infant PrEP to prevent mother-to-child transmission, in combination or even as a sole preventive regimen. ${ }^{16-19}$ The most recent of these, the ANRS 12174 trial, showed that infant prophylaxis with either lamivudine (3TC) or boosted lopinavir (LPV/r) daily throughout breastfeeding for up to 12 months among infants of HIV infected women who did not qualify for ART for their own health was well tolerated and reduced the risk of postnatal transmission at 1 year of age to $0.5 \%$ (per protocol) or $1.4 \%$ (intention to treat). ${ }^{19}$ Adherence to infant PrEP in the trial was particularly high (over $90 \%) .^{19}$

Pharmacological data suggest that plasma drug levels lower than the therapeutic threshold are sufficient to protect infants. ${ }^{20}$ In addition, pharmacokinetic studies in infants breastfed by mothers taking ART show that their antiretroviral drug plasma levels are largely below $5 \%$ of the therapeutic level. ${ }^{21}$ This suggests that infant PrEP could be combined with maternal ART without a risk of overdosing or cumulative adverse effects.

In the near future, injectable long acting antiretroviral drugs such as rilpivirine or cabotegravir may become available. This would enable PrEP to be started from birth with only a few additional administrations to cover the duration of breastfeeding. The estimated cost of daily administration lamivudine paediatric suspension in a breastfed infant is less than $\$ 15$ (£12; €14) a year. Cost effectiveness studies of infant PrEP have not been done, but the low cost of the infant PrEP regimen suggests that the expected benefit would justify the expense of adding it to maternal ART. Indeed, even if only one HIV infection was averted out of 100 exposed infants, the cost per averted infection would be minimal $(\$ 1500)$.

\section{When should infant PrEP be recommended?}

Infant PrEP should certainly be advised when the mother's HIV infection is untreated or if she has a detectable viral load despite ART. Such situations can occur when the mother does not want or is unable to take ART or is at high risk of poor drug adherence. The determinants of maternal adherence to ART probably differ from those for adherence to infant PrEP. Unpublished data collected during the ANRS 12174 trial suggest that most pregnant or lactating mothers prefer to administer a prophylactic antiretroviral drug to their exposed infant than to adhere to their own ART. However, this targeted approach may be seen as complex and hampered by programmatic problems in some settings. A simpler alternative would be to protect all HIV exposed infants with PrEP during the breastfeeding period, on the basis that the PrEP drugs are safe and that optimal maternal adherence to ART in the perinatal period cannot be assumed. Of course, treatment of the mother should remain a priority.

\section{Conclusion}

Mother-to-child HIV transmission among breastfed infants is not unlike HIV transmission associated with discordant couples, with the mother and child having frequent contact that exposes the infant to HIV, even if the mother is provided with a 
suppressive ART regimen. Given the evidence that infant PrEP is effective, there is a moral imperative to correct the policy inequity that exists between HIV exposed adults and children. Scaling up existing interventions and extended access to PrEP to those most in need are the most cost effective ways to stem new HIV infections. ${ }^{22}$ Expanding global prevention guidelines to include infant PrEP for infants exposed to HIV by breast feeding could be a major breakthrough as a public health approach to eliminate mother-to-child transmission.

Contributors and sources: This article is based on recent publications and conference presentations on PMTCT. All authors conceptualised this article during meetings on mother and child health. PV wrote the first draft of the manuscript and coordinated the revised versions. All authors reviewed and approved the final version and are responsible for the final content of the manuscript.

Provenance and peer review: Not commissioned; externally peer reviewed.

Competing interests: We have read and understood BMJ policy on declaration of interests and have no relevant interests to declare.

1 Grant RM, Lama JR, Anderson PL, et al. iPrEx Study Team. Preexposure chemoprophylaxis for HIV prevention in men who have sex with men. $N$ Engl J Med 2010:356:2587-99. doi:10.1056/NEJMoa1011205 pmid:21091279.

2 Molina JM, Capitant C, Spire B, et al. ANRS IPERGAY Study Group. On-demand preexposure prophylaxis in men at high risk for HIV-1 infection. N Engl J Med 2015;356:2237-46. doi:10.1056/NEJMoa1506273 pmid:26624850.

3 WHO. WHO technical update on pre exposure prophylaxis (PreP). February 2015. http:/ lapps.who.int/iris/bitstream/10665/153587/1/WHO HIV 2015.4 eng.pdf

4 Price JT, Wheeler SB, Stranix-Chibanda L, et al. Cost-effectiveness of pre-exposure HIV prophylaxis during pregnancy and breastfeeding in Sub-Saharan Africa. J Acquir Immune Defic Syndr 2016;356(Suppl 2):S145-53. doi:10.1097/QAl.0000000000001063 pmid: 27355502.

5 WHO. Consolidated guidelines on the use of antiretroviral drugs for treating and preventing HIV infection. Recommendations for a public health approach. 2nd ed. 2016. http://apps. who.int/iris/bitstream/10665/208825/1/9789241549684_eng.pdf

6 UNAIDS. 2016 prevention gap report. http://www.unaids.org/sites/default/files/media_ asset/2016-prevention-gapreport_en.pdf

7 Mofenson LM. State of the art in prevention of mother-to-child transmission. 2nd workshop on prevention trials in infants born to HIV-positive mothers, Maputo, Mozambique, 5-7 Feb 2017.

8 Maman D, Huerga $\mathrm{H}$, Mukui I, et al. Most breastfeeding women with high viral load are still undiagnosed in sub Saharan Africa. Abstract 32. Conference on retroviruses and opportunistic infections, Seattle, Washington, 23-26 Feb 2015.
9 Tenthani L, Haas AD, Tweya $\mathrm{H}$, et al. Ministry of Health in Malawi and leDEA Southern Africa. Retention in care under universal antiretroviral therapy for HIV-infected pregnant and breastfeeding women ('Option B+') in Malawi. AIDS 2014;356:589-98. doi:10.1097/ QAD.0000000000000143 pmid:24468999.

10 Abrams EJ, Langwenya N, Gachuhi A, et al. Impact of Option B+ on ART uptake and retention in Swaziland: a stepped-wedge trial. Abstract 34. Conference on retroviruses and opportunistic infections, Boston, MA, 22-25 Feb 2016.

11 Manigart O, Crepin M, Leroy V, et al. Diminution de la Transmission Mere-Enfant Study Group. Effect of perinatal zidovudine prophylaxis on the evolution of cell-free HIV-1 RNA in breast milk and on postnatal transmission. $J$ Infect Dis 2004;356:1422-8. doi:10.1086/ 424569 pmid: 15378434.

12 Van de Perre P, Rubbo PA, Viljoen J, et al. HIV-1 reservoirs in breast milk and translational challenges to elimination of breast-feeding transmission of HIV-1. Sci Trans/ Med 2012;356:143sr3. doi:10.1126/scitransImed.3003327 pmid:22814853.

13 Rollins N, Mahy M, Becquet R, Kuhn L, Creek T, Mofenson L. Estimates of peripartum and postnatal mother-to-child transmission probabilities of HIV for use in Spectrum and other population-based models. Sex Transm Infect 2012;356(Suppl 2):i44-51. doi:10. 1136/sextrans-2012-050709 pmid:23172345.

14 WHO, UNICEF. Guideline updates on HIV and infant feeding. $2016 \mathrm{http}: / /$ apps.who.int/ iris/bitstream/10665/246260/1/9789241549707-eng.pdf

15 Connor EM, Sperling RS, Gelber R, et al. Reduction of maternal-infant transmission of human immunodeficiency virus type 1 with zidovudine treatment. Pediatric AIDS Clinical Trials Group Protocol 076 Study Group. N Engl J Med 1994;356:1173-80. doi:10.1056/ NEJM199411033311801 pmid:7935654.

16 Kilewo C, Karlsson K, Massawe A, et al. Mitra Study Team. Prevention of mother-to-child transmission of HIV-1 through breast-feeding by treating infants prophylactically with lamivudine in Dar es Salaam, Tanzania: the Mitra Study. J Acquir Immune Defic Syndr 2008:356:315-23. doi:10.1097/QAI.0b013e31816e395c pmid:18344879.

17 Kumwenda NI, Hoover DR, Mofenson LM, et al. Extended antiretroviral prophylaxis to reduce breast-milk HIV-1 transmission. N Engl J Med 2008;356:119-29. doi:10.1056/ NEJMoa0801941 pmid:18525035.

18 Coovadia HM, Brown ER, Fowler MG, et al. HPTN 046 protocol team. Efficacy and safety of an extended nevirapine regimen in infant children of breastfeeding mothers with HIV-1 infection for prevention of postnatal HIV-1 transmission (HPTN 046): a randomised, double-blind, placebo-controlled trial. Lancet 2012;356:221-8. doi:10.1016/S0140-6736( 11)61653-X pmid:22196945.

19 Nagot N, Kankasa C, Tumwine JK, et al. ANRS 12174 Trial Group. Extended pre-exposure prophylaxis with lopinavir-ritonavir versus lamivudine to prevent HIV-1 transmission through breastfeeding up to 50 weeks in infants in Africa (ANRS 12174): a randomised controlled trial. Lancet 2016;356:566-73. doi:10.1016/S0140-6736(15)00984-8 pmid:26603917.

20 Foissac F, Blume J, Tréluyer JM, et al. ANRS 12174 Trial Group. Are prophylactic and therapeutic target concentrations different? The case of lopinavir/ritonavir or lamivudine administered to infants for the prevention of mother-to-child HIV-1 transmission during breastfeeding. Antimicrob Agents Chemother 2017;356:e1869-16.pmid:27895016.

21 Shapiro RL, Rossi S, Ogwu A, et al. Therapeutic levels of lopinavir in late pregnancy and abacavir passage into breast milk in the Mma Bana Study, Botswana. Antivir Ther 2013;356:585-90. doi:10.3851/IMP2474 pmid:23183881.

22 Smith JA, Anderson SJ, Harris KL, et al. Maximising HIV prevention by balancing the opportunities of today with the promises of tomorrow: a modelling study. Lancet HIV 2016;356:e289-96. doi:10.1016/S2352-3018(16)30036-4 pmid:27365203.

\section{Accepted: 10022017}

Published by the BMJ Publishing Group Limited. For permission to use (where not already granted under a licence) please go to http://group.bmj.com/group/rights-licensing/ permissions 


\section{Key messages}

WHO recommends pre-exposure prophylaxis for any group with an expected incidence of HIV infection above $3 / 100$ person-years Current strategies to prevent mother-to-child transmission of HIV cover only the first six weeks

Many infections of breastfed infants occur after this period

Adding infant PrEP to maternal ART is cheap and does not expose infants to unsafe doses

Routine infant PrEP has the potential to be a breakthrough in elimination of mother-to-child transmission 